Kaum Fortschritte bei der Verhütung für Männer

\title{
Die „Pille“ für ihn bleibt auch auf lange Sicht nur ein frommer Wunsch
}

\author{
Die „Pille für den Mann“ - sie wird es so schnell nicht geben. Die umständliche \\ Applikation per Spritze und ein hoher Anteil von Nonrespondern machen der \\ Forschung zu schaffen.
}

_ Über die „Pille für den Mann“ wird schon so lange geredet, wie es sie für die Frau gibt, aber bislang hat noch kein einziges pharmakologisches Kontrazeptivum für den Mann den Durchbruch geschafft. Die Gründe sind vielfältig: Nebenwirkungen, eine umständliche Anwendung, häufige Kontrollen und die lange Zeit bis zur wirksamen Unterdrückung der Spermatogenese, so Reproduktionsmediziner um Dr. Mara Roth von der Universität in Seattle.

Aufgrund der physiologischen $\mathrm{Zu}$ sammenhänge sollte sich Testosteron prima als Kontrazeptivum eignen. Bereits in den 1980er- und 1990er-Jahren gab es hierzu zwei wegweisende Studien der WHO: $200 \mathrm{mg}$ Testosteron-Enantat, jede Woche intramuskulär injiziert, führten bei zwei Drittel der Männer zur einer Azoospermie. Außerdem konnte gezeigt werden, dass eine Oligozoospermie mit Spermienkonzentrationen unter 1 Million/ml relativ sicher ist (Schwangerschaftswahrscheinlichkeit von rund $1 \%$ pro Jahr).

Bei der Verhütung hat er nur eine Wahl

- das Kondom.

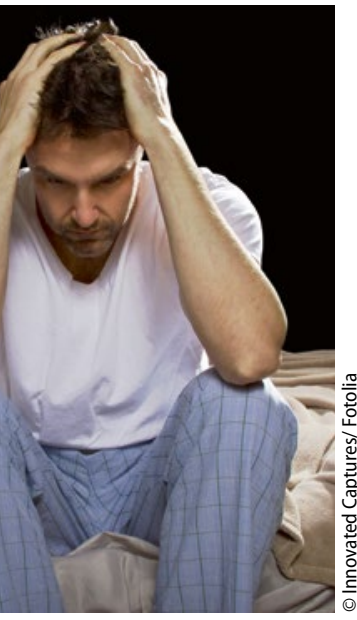

\section{Wöchentliche Injektionen}

Testosteron kam als Verhütungsmittel dennoch nicht auf den Markt, unter anderem weil es vier Monate dauerte, bis die Spermatogenese ausreichend unterdrückt war. Verkürzen lässt sich die Zeit bis zur Oligozoospermie mithilfe von Progestinen - Gestagenderivaten wie sie auch zur Empfängnisverhütung bei Frauen verwendet werden. Gleichzeitig kann damit die Testosterondosis gesenkt und Nebenwirkungen wie Akne und Haarverlust vermindert werden.

In einer Studie mit Testosteron und Etonogestrel als Implantat wurden je nach Dosierung Oligozoospermieraten um $90 \%$ erreicht. Allerdings konnten Nebenwirkungen der beiden Präparate nicht klar voneinander abgegrenzt werden, außerdem muss regelmäßig Testosteron injiziert werden. Die Injektionsfrequenz lässt sich zwar mit lang wirksamen Formulierungen wie Testosteron-Undecanoat auf einmal alle drei Monate begrenzen, dennoch dürfte eine Kombinationstherapie mit Implantaten und regelmäßigen Injektionen kaum Chancen auf dem Markt haben.

Besser könnte es für eine Gel-Kombination aussehen: In einer Studie unterschritten $89 \%$ der Männer nach 20 Tagen Anwendung von Testosteron- und Nestoron-Gel die Grenzkonzentration von 1 Million Spermien/ml, und das Gestagen Nestoron zeigte keine androgene, östrogene und kortikoide Wirkung.

\section{Das Problem der Pille: Nonresponse}

Auch das synthetische Androgen Dimethandrolon-Undecanoat (DMAU) wird derzeit klinisch geprüft. Der Vorteil: Es lässt sich als Pille schlucken. Phase-IDaten deuten auf eine gute und reversible

\section{Theoretisch ideal: Testosteron}

Bei einem gesunden zeugungsfähigen Mann führt Gonadotropin-releasing Hormon (GnRH) aus dem Hypothalamus zur Freisetzung von luteinisierendem Hormon (LH) und follikelstimulierendem Hormon (FSH) aus der Hypophyse. LH führt zur Testosteronausschüttung in den Leydigzellen des Hodens. Testosteron zusammen mit FSH kurbelt die Spermatogenese in den Sertolizellen an, wirkt gleichzeitig aber als Bremse in Hypothalamus und Hypophyse: Hohe Testosteronspiegel blockieren die LH- und FSH-Freisetzung und damit die Spermatogenese.

Unterdrückung der Spermatogenese. Doch bei etwa $10-15 \%$ der Männer klappt dies nicht ausreichend. Weshalb, ist noch unklar, und damit fehlen bislang auch Marker, um Responder und Nonrespondern im Voraus zu unterscheiden. Ein weiteres Problem: Wenn ein Präparat geschluckt und das andere gespritzt werden muss, ist die Gefahr hoch, dass eines von beiden gelegentlich vergessen wird. Dies könnte dann die Spermatogenese über Wochen hinweg wieder ankurbeln.

Nicht zuletzt aus solchen Gründen habe das Interesse der Industrie an der „Pille für den Mann“ drastisch abgenommen, so die Wissenschaftler um Roth. Es würden kaum noch Gelder in die Entwicklung solcher Kontrazeptiva gesteckt. Auch die Sicherheitsrisiken scheinen vielen Unternehmen zu hoch - die Gefahr, an den hohen Hürden der Zulassungsbehörden zu scheitern, sei enorm.

Thomas Müller

- Roth MY et al. Male hormonal contraception: looking back and moving forward. Andrology 2015, online 9. Oktober; doi: 10.1111/andr.12110, http://dx.doi.org/10.1111/andr.12110 